Article

\title{
Long-Term Changes in Pain Sensitivity in an Animal Model of Social Anxiety
}

\section{Alessandra Berry ${ }^{\dagger} *$, Veronica Bellisario ${ }^{\dagger}$, Sara Capoccia, Nadia Francia, Enrico Alleva and Francesca Cirulli}

Section of Behavioural Neurosciences, Department of Cell Biology and Neurosciences, Istituto Superiore di Sanità, Viale Regina Elena 299, Rome 00161, Italy;

E-Mails: veronica.bellisario@guest.iss.it (V.B.); sara.capoccia@guest.iss.it (S.C.); nadia.francia@iss.it (N.F.); enrico.alleva@iss.it (E.A.); francesca.cirulli@iss.it (F.C.)

$\dagger$ These authors contributed equally to this work.

* Author to whom correspondence should be addressed; E-Mail: alessandra.berry@iss.it; Tel.: +39-06-4990 (ext. 2480); Fax: +39-06-495-7821.

Received: 27 March 2014; in revised form: 23 June 2014 / Accepted: 25 June 2014 /

Published: 2 July 2014

\begin{abstract}
Animal models with an eco-ethological relevance can help in identifying novel and reliable stress-related markers. To this end, 3-month-old C57BL/6J male mice were exposed to social defeat (SD) stress for 10 days as this stressor shows good face and predictive validity for several models of human affective disorders including depression, social phobia and post-traumatic stress disorder. Social avoidance and pain threshold were assessed $24 \mathrm{~h}$ and 4 weeks after the end of SD stress, while corticosterone was assayed at the beginning and at the end of the stressful procedure (days 1 and 10). SD subjects were characterized by increased corticosterone levels (30 $\mathrm{min}$ following stress exposure), increased latency to approach the social target in the short-term as well as increased emotionality in the long-term. Moreover, an increase in nociceptive threshold (stress-induced analgesia) was found both in the short-term and 4 weeks after the end of stress. These data indicate that the SD paradigm is able to induce emotional changes associated with a stressful/traumatic event. In addition, they indicate that variations in the nociceptive threshold might represent a physiological marker of both short- and long-term effects of stress.
\end{abstract}


Keywords: mice; animal model; stress-induced analgesia; pain sensitivity; social defeat stress; emotionality

\section{Introduction}

Animal models represent a key heuristic approach to identifying reliable markers and pathophysiological aspects of stress-related diseases, such as generalized anxiety, major depression, post-traumatic stress disorder (PTSD) and schizophrenia [1-5]. In an attempt to model pathologies associated with stress, powerful stressors such as cold, electric foot-shock, forced swimming, restraint and chronic unpredictable stress are commonly used. However, as many authors have pointed out, most of these stressful challenges used in the laboratory setting are not so relevant to situations that humans encounter in their everyday life, likewise they hardly resemble those usually faced by rodents in a natural context [6-9]. By contrast, social stressors are effective in triggering powerful physiological and, more importantly, emotional responses both in humans and in social animals such as rats and mice [5,10-15] also leading to avoidance/withdrawal, a behavioral trait which is common in several human affective disorders including depression, social phobia and also PTSD [16]. In particular, it has been proposed that, compared to models based on physical stressors, social defeat (SD) stress, which (in mice and rats) is based on the experimental induction of social conflicts through repeated exposures to a larger, dominant, conspecific might represent a reliable paradigm to model stress-induced human psychopathological traits [17]. Indeed, SD stress does not result in habituation upon repeated presentations, providing an ethologically and ecologically relevant form of persistent emotional distress [18]. Chronic exposure to this kind of stress profoundly alters the motivation for social interactions in rodents [19-21] and also leads to short- and long-term behavioral and physiological changes, such as decreased locomotion and exploratory activity [22,23], in addition to increased emotionality [10,24]. Berton and colleagues, using a modified version of the SD stress in mice, observed long-term effects on neural and behavioral plasticity in response to aversive social experiences including social avoidance up to 4 weeks from the end of the stressful procedure [16]. Intriguingly, these authors also showed that chronic treatment with antidepressants is able to normalize social withdrawal in this mouse model. Thus, SD stress appears as a solid animal model with good face and predictive validity that may help in identifying novel and reliable markers of stress-related psychosocial alterations.

Psychiatric pathologies in which stressful/traumatic events play a main role in their etiology, such as PTSD acute stress and borderline personality disorders (BPD), are characterized by common stress-induced features, such as intrusive memories and dissociative states, involving numbing and changes in pain perception [25-27]. A well characterized physiological response to stress is an increase in nociceptive threshold that relies upon the activation of different endogenous opioid and/or non-opioid pain inhibitory systems [28-32]. This phenomenon is commonly known as stress-induced analgesia (SIA) [33] and represents a key component of the organism's defense system against aversive stimuli, preparing for the "fight or flight" response [34]. Former experiments on social conflict have provided a biologically relevant model of SIA with decreased nociception in defeated 
mice [21,35-38]. However, current knowledge of this aspect of nociception refers mostly to acute responses occurring within a few hours from the end of stress exposure. Jackson and co-workers, in 1979 , were the first to show that $24 \mathrm{~h}$ after exposure to electric foot shock rats still show an analgesic reaction [39].

The aim of this study was to compare the short- $(24 \mathrm{~h})$ and the long-term (4 weeks) effects of exposure to a chronic psychosocial stress (SD) on social anxiety with a main focus on specific changes in pain sensitivity resulting from SD. We hypothesized that defeated mice would show greater social avoidance and higher pain threshold than controls. In order to test this hypothesis, we investigated specific behavioral changes as a result of the social defeat experience (by means of the social avoidance test-SAT) and the nociceptive response (by means of the hot plate test) $24 \mathrm{~h}$ and, for the first time, 4 weeks after the end of SD. Physiological parameters, such as changes in plasma levels of corticosterone (CORT) and body weight, were assessed before and at the end of the 10-day stressful procedure to control for SD effectiveness.

\section{Experimental Section}

Animals: experimental subjects were male mice either from a C57BL/6J ( $n=26$; SD or control subjects-CTRL) or CD-1 ( $n=32$; aggressive resident mice) strain purchased from a commercial breeder (Charles River, Calco, Italy). Upon arrival, C57BL/6J mice were pair-housed in cages $(37 \times 21 \times 19 \mathrm{~cm})$ provided by a perforated Plexiglas partition allowing sensory but not physical contact, meaning they were housed in two compartments $(18.5 \times 21 \times 19 \mathrm{~cm})$ within the same cage, separated by a perforated partition wall for 7 days (acclimatization); CD-1 mice were single-housed. Animals were all kept in the same room provided by air conditioning (temperature $21 \pm 1{ }^{\circ} \mathrm{C}$, relative humidity $60 \% \pm 10 \%$ ) under a reversed $12 / 12 \mathrm{~h}$ light/dark cycle with lights off from 06:30 a.m. to 06:30 p.m. Pellet food (standard diet Altromin-R, purchased from Rieper, Italy) and tap-water were continuously available.

Following 1 week of acclimatization, experimental subjects were randomly assigned to the chronic social defeat condition ( $\mathrm{SD}, n=14 ; 10$ days of SD stress) or to a control group (CTRL, $n=12$; daily handling). SD mice were blood sampled by tail nick at the beginning (day 1) and at the end of the stressful procedure (day 10) to assess neuroendocrine activation. At the end of the 10-day stress paradigm, all subjects were tested for social avoidance (SAT) and nociceptive threshold (hot plate (HP) test) either under short- (24 h) or long-term (4 weeks) conditions (final number of subjects for each group: CTRLs, $n=6$ both for short- and long-term conditions; SD, $n=7$ both for short- and long-term conditions); see Figure 1 for a schematic representation of the experimental timeline. Behavioral performances were video-recorded and the behavioral analysis was carried out from the videotape, using commercial software ("The Observer 3.0"). At the end of each session, apparatuses were cleaned by cotton pads soaked with a $50 \%$ solution of ethanol and water. Since it has been shown that the effects of repeated psychosocial stress are more pronounced if the test is performed during the active circadian phase of mice [40], all tests were conducted between 9:30 a.m. and 12:30 p.m. under dim light (1 lux), during the dark period of a reversed light cycle. 
Figure 1. Schematic representation of the experimental timeline. The experiment lasted 38 days in total. SD subjects underwent 10 days of daily bouts of social defeat, followed by continuous protected sensory contact with their aggressor. Body weight was assessed on the day before stress took place (day 0), on day 5 and on day 10 of SD. Blood samples for corticosterone assessment were collected on days 1 and 10. SD and CTRL subjects were either tested on day 11 (24 h following the end of the SD procedure, short-term effects of stress), or on day 38 (4 weeks following the end of the SD procedure, long-term effects of stress). b.w.: body weight; CORT: corticosterone; SAT: Social Avoidance Test; HP: Hot Plate Test; SD: Social Defeat.

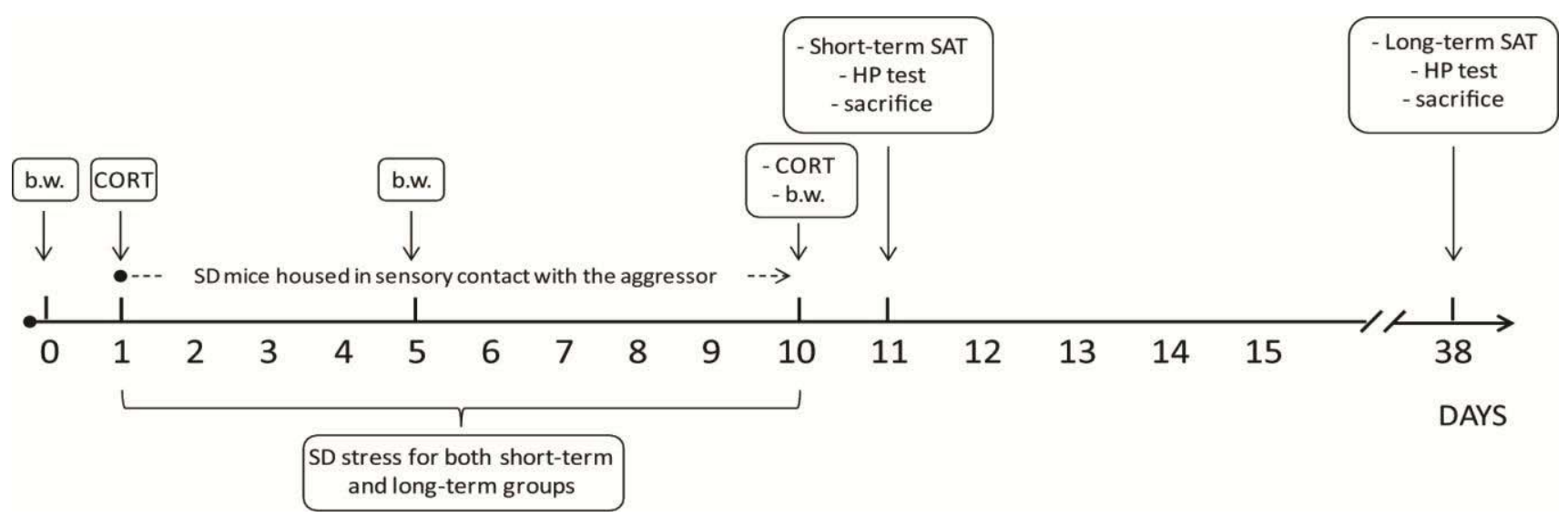

Animal handling and experimental procedures were performed in accordance with the Guidelines laid down by the European Communities Council Directive 2010/63/EU and with the Italian legislation on animal experimentation (Decreto L.vo 116/92).

Social Defeat Stress: C57BL/6J mice were randomly assigned to the chronic social defeat condition (SD, $n=14 ; 10$ days of SD stress) or to a control group (CTRL, $n=12$; daily handling). SD subjects underwent 10 days of daily bouts of social defeat, followed by continuous protected sensory contact with their aggressor $[13,16]$. More precisely, each SD mouse $(\mathrm{C} 57 \mathrm{BL} / 6 \mathrm{~J}, n=14)$ was introduced into the home cage of an unfamiliar resident $(\mathrm{CD}-1, n=14)$ for 5 min and was physically defeated, soon after, residents and intruders were physically separated by a perforated transparent Plexiglas partition and maintained in sensory contact for $24 \mathrm{~h}$ until the next trial. Every day, each SD mouse was exposed to a different aggressor while CTRL subjects were housed in stable pairs with a same strain conspecific, under the same sensory conditions, and were daily handled by the time SD subjects underwent social stress. This housing was maintained throughout the study and was meant to avoid any bias related to changes in the housing conditions after the stressful procedure in the SD group. At the end of the 10 days of stress, SD mice were pair-housed with a same sex, same strain conspecific in a cage provided by a Plexiglas perforated partition for either $24 \mathrm{~h}$ or 4 weeks (28 days). Since C57BL/6J mice are usually smaller than same-age CD-1 conspecifics, in order to prevent excessive physical aggression possibly leading to a lethal outcome, intruders (C57BL/6J) were selected so to have a body size comparable to that of residents (CD-1). Resident mice were selected for their attack latencies reliably shorter than $30 \mathrm{~s}$ upon 3 consecutive screening tests [16]. CD-1 mice were housed alone at least 3 weeks prior to the SD stress procedure in order to increase aggressive behavior. 
The intruder mouse was considered "defeated" when displaying submissive postures. Excessive aggressive behaviors (e.g., incessant biting that could lead to injuries) were interrupted by inserting the Plexiglas partition.

Body weight: to assess stress-related changes in body weight, SD mice were weighed the day before the stress started (day 0) and on days 5 and 10 of the SD procedure. The body weight of control subjects was also registered on the same days, i.e., on handling days 0,5 and 10.

Neuroendocrine activation: to assess the effect of the SD stress on hypothalamic-pituitary-adrenal (HPA) axis activation, plasma CORT levels were measured on the first (day 1) and on the last day (day 10) of the procedure at different time points, i.e., soon before (0 $\mathrm{min})$ and following (30 and $120 \mathrm{~min}$ ) the defeat. Blood sampling was performed by means of the tail nick a standard minimally-invasive consolidated procedure in use in our laboratory and many others. Briefly: a mouse is taken from the cage and gently placed on the metal top of a clean cage; the experimenter holds the tip of the tail while the mouse that is not restrained is allowed to move. By means of a razor blade, a small nick is performed (approximately $2 \mathrm{~mm}$ wide $\times 0.5 \mathrm{~mm}$ deep) in the tail, perpendicular to the tail vein, approximately $2 \mathrm{~cm}$ from the tip of the tail. Blood droplets are directly collected into EDTA-coated capillary tubes. For further details on the procedure, see also [41,42]. To prevent bleeding before the test took place, the animals' tail was carefully dried and pinched for $10 \mathrm{~s}$ with a clean tissue.

Social Avoidance Test (SAT): social avoidance behavior is a widely used indicator of the effects of social stress, with defeated subjects decreasing the time spent investigating a novel conspecific [16]. Thus, to assess the short- $(24 \mathrm{~h})$ and long-term (4 weeks) effects of the exposure to a socially stressful event, experimental subjects (CTRL, $n=6$ and $\mathrm{SD}, n=7$ for both short- and long-term assessment) underwent a SAT. More precisely, mice were introduced to a plastic open field arena $(42 \times 42 \mathrm{~cm})$ for two consecutive $2.5 \mathrm{~min}$ sessions (a test duration chosen for its optimal sensitivity/throughput ratio [16]). During session I ("no target"), the open field contained an empty wire mesh cage (diameter $=10 \mathrm{~cm}$, $\mathrm{h}=10 \mathrm{~cm}$ ) located in the middle of one side of the arena. During session II ("social target"), a social target (an unfamiliar CD-1 male mouse) was introduced into the wire mesh cage. Between the two sessions, the experimental subject was placed back into its home cage for $1 \mathrm{~min}$. The time spent in the "interaction zone" ( $8 \mathrm{~cm}$ wide corridor surrounding the cage) vs. the "corners" (opposite to the location of the cage) was scored as a reliable measure of social avoidance. When data were analyzed, the arena was ideally divided into squares $(7 \times 7 \mathrm{~cm})$ and latency and frequency of locomotion (crossings of squares) were scored, in addition to latency, frequency and duration of the risk assessment behavior (stretched-attend posture-SAP), freezing and exploration of the wire mesh cage (touching object).

Behaviors were defined as follows: crossing, crossing the square limits with all paws; stretched-attend posture, exploratory posture in which the body is stretched forward and then retracted to the original position without any forward locomotion; freezing, self-explanatory; touching object, self-explanatory.

Hot Plate Test (HP): two hours following the end of the SAT, subjects were placed on a hot metal plate set at $50 \pm 1{ }^{\circ} \mathrm{C}$ enclosed by a $19 \mathrm{~cm}$ diameter Perspex cylinder (Model D837; Basile, Socrel Comerio, Italy), to prevent animals from jumping off the plate. Although many studies use higher temperatures, we have previously shown that already at $50 \pm 1{ }^{\circ} \mathrm{C}$ it is possible to test differences in the sensitivity to a thermal noxious stimulus both in mice and rats by overall reducing suffering of experimental subjects $[43,44]$. Nociception was assessed by scoring latency, frequency and duration of 
forepaw and hindpaw licking, the latter behavioral item (hindpaw licking) being the most reliable measure of pain sensitivity [44]. A cut-off time of $60 \mathrm{~s}$ was used.

Radioimmunoassay for corticosterone determination (RIA): Blood samples (100 $\mu \mathrm{L}$, approximate volume) were collected individually in potassium EDTA coated tubes (1.6 mg EDTA/mL blood; Sarstedt, Germany). All samples were kept on ice and later centrifuged at $3000 \mathrm{rpm}$ for $15 \mathrm{~min}$ at $+4{ }^{\circ} \mathrm{C}$. Blood plasma was transferred to Eppendorf tubes for CORT determination and stored at $-80{ }^{\circ} \mathrm{C}$ until further analysis. CORT was measured using commercially available radioimmunoassay (RIA) kit (MP Biomedicals, LLC, NY, USA) containing 125 iodine labelled CORT; $5 \mu \mathrm{L}$ of plasma were sufficient to carry out CORT measurement. Sensitivity of the assay was $0.125 \mathrm{mg} / \mathrm{dL}$, inter- and intra-assay variation was less than $10 \%$ and $5 \%$, respectively. Vials were counted for $2 \mathrm{~min}$ in a gamma-scintillation counter (Packard Minaxi Gamma counter, Series 5000).

Statistical analysis: Data were analyzed using parametric analysis of variance (ANOVA) with group (stress (SD) vs. control (CTRL)) as between-subjects factor and days, minutes, sessions ("no target" vs. "social target") and zones ("center" vs. "corners" vs. "interaction zone") as within-subject repeated measures, when appropriate (body weight measurement, CORT assessment and SAT). Since subjects tested at $24 \mathrm{~h}$ and at 4 weeks belonged to two different groups of animals, we decided to run separate statistical analyses. Post hoc comparisons were performed using the Tukey's test. Data are expressed as mean + SEM. A significance level of 0.05 was chosen.

\section{Results and Discussion}

\subsection{Body Weight}

The SD paradigm affected body weight of the experimental subjects. In fact, on day 10 SD mice showed a significant decrease in body weight compared to CTRL subjects (interaction between days and stress condition: $F_{2,48}=4.176, p=0.0213$, see Figure 2).

Figure 2. Body weight. Social defeated (SD) subjects decreased their body weight following 10 days of chronic SD stress. Data are presented as mean + S.E.M. (CTRL, $n=12$; $\mathrm{SD}, n=14) . * *<0.01$.

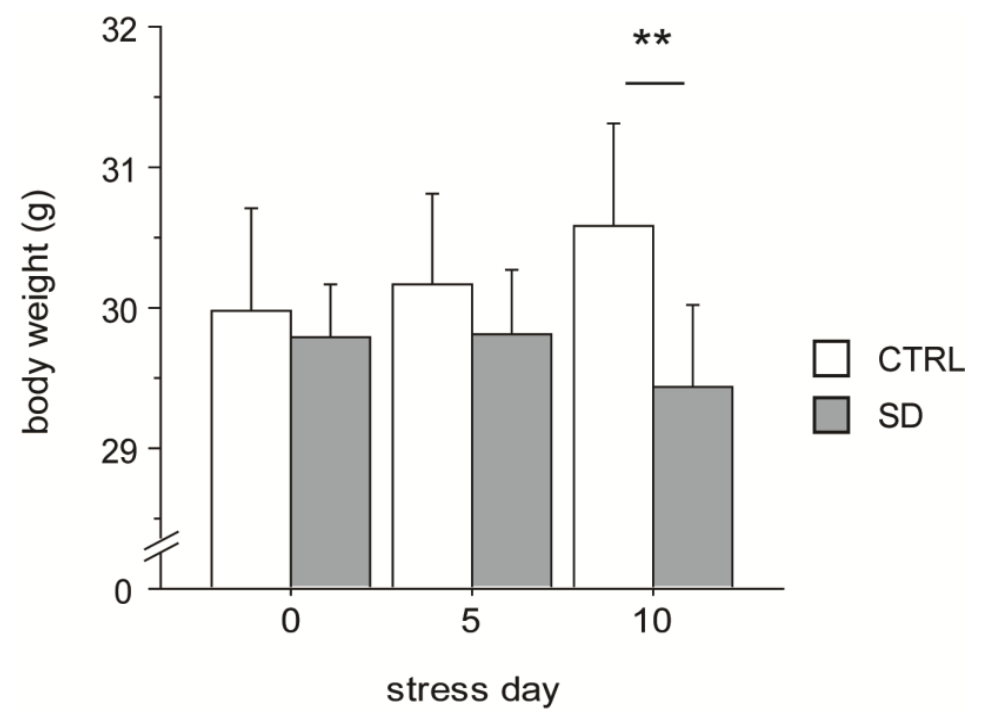




\subsection{Neuroendocrine Activation}

The social defeat paradigm was effective in challenging the HPA axis (main effect of repeated measures: $\left.F_{2,26}=78.342, p<0.0001\right)$. In particular, the SD procedure leads to an overall increase in CORT levels $30 \mathrm{~min}$ from the onset of stress (basal vs. 30, $p<0.01$ ) and is followed by a smooth decrease $120 \mathrm{~min}$ later (30 vs. $120 \mathrm{~min}, p<0.05$ ). No difference was found between day 1 and day 10 that were overall characterized by a similar profile in the HPA axis activation during time (interaction between days and time course: $F_{2,26}=0.921, p=0.4106$, see Figure 3). CORT levels were not assessed in the CTRL group thus we cannot exclude that, although minimally-invasive, the blood sampling procedure might have contributed to the observed HPA axis activation.

Figure 3. Neuroendocrine activation following the first (day 1) and the last encounter (day 10). SD stress is able to challenge the HPA axis. Experimental subjects do not show a significant habituation to the stressful procedure following 10 days of SD, being characterized by a similar neuroendocrine profile on day 1 and on day 10. This lack of habituation is due to the fact that each day a different social stimulus was used, thus preventing a clear habituation profile. Data are presented as mean + S.E.M. (SD, $n=14)$. $* * p<0.01$, time point 0 vs. both 30 and $120 \mathrm{~min} ;{ }^{*} p<0.05,30 \mathrm{~min} v s .120 \mathrm{~min}$.

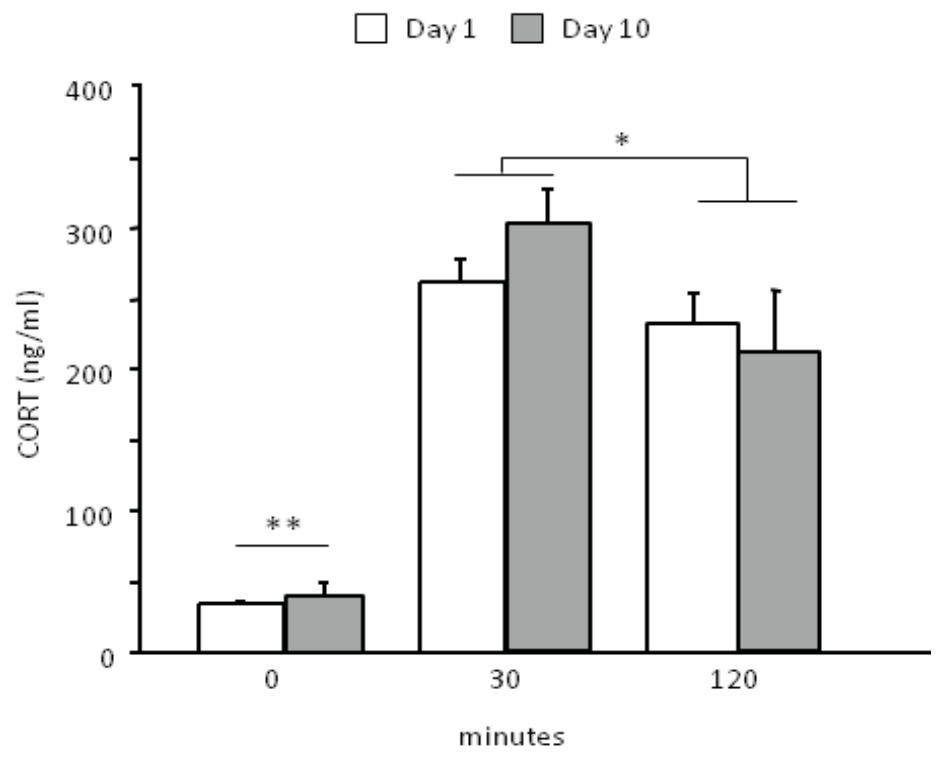

\subsection{Social Avoidance Test-SAT}

\subsubsection{Short-Term Effects $(24 \mathrm{~h})$}

Analysis of sessions I and II of the SAT indicated that the behavior of SD mice was affected by previous exposure to a stressful experience since they showed a higher latency to touch the wire mesh cage when the social stimulus was present (interaction between stimulus and stress condition for touching object behavior: $F_{1,11}=10.521, p=0.0078$, see Figure 4A). By contrast, no difference was found in the time spent in the "interaction zone" between SD and CTRL mice (main effect of stress condition: $F_{1,11}=0.658, p=0.4343$, i.e., average time spent either in the presence or in the absence of the social target, see Figure 4B). 
Figure 4. Social Avoidance Test (SAT). The presence of a social stimulus increased the latency of SD mice to touch the wire mesh cage (touching object) 24 h (A) but not 4 weeks after the end of the stress procedure $(\mathbf{E})$. Overall, independently from the presence of the social stimulus, in the long-term situation (4 weeks), SD subjects spent less time in the interaction zone than CTRLs $(\mathbf{F})$, showed both a shorter latency to perform $S A P(\mathbf{G})$ and to freeze $(\mathbf{H})$. No difference was found between SD and CTRL mice in the short-term condition (B, $\mathbf{C}$ and $\mathbf{D}$, respectively). Data are presented as mean + S.E.M. $n=7$ (SD) and 6 (CTRL). Subjects were tested only once either at $24 \mathrm{~h}$ or 4 weeks from the end of SD. $* p<0.05 . * * p<0.01$ (main effect of the social condition).
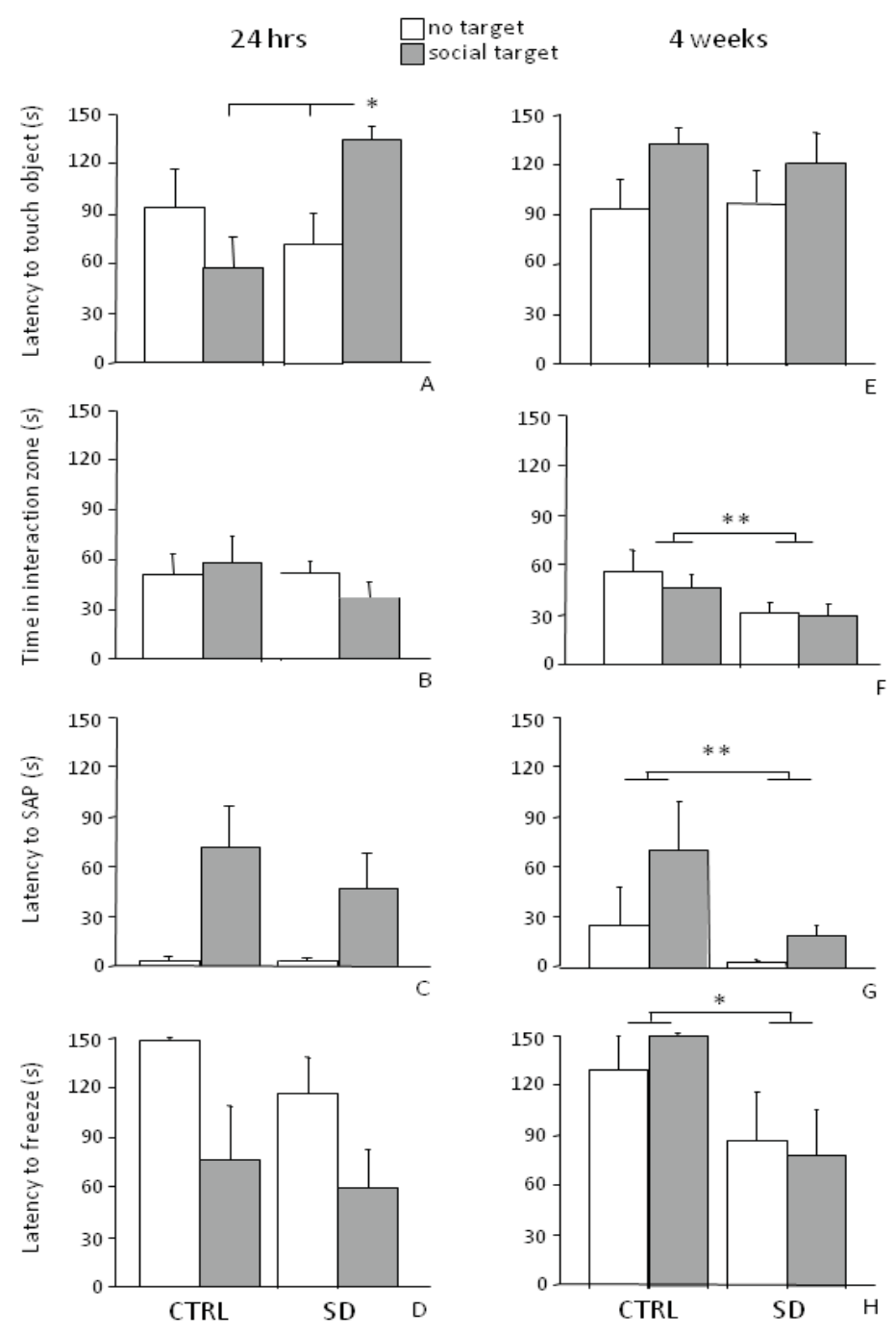

In general, all mice showed an increased locomotor activity in the interaction zone, i.e., in the proximity of the wire mesh cage (main effect of the zone: $F_{2,22}=20.863, p=0.0001$, post hoc comparisons: "interaction zone" both $v s$. "center" and "corners", $p<0.01)$. All subjects, independently from CTRL or SD conditions, showed a decrease in locomotor activity (crossing frequency) when the social stimulus was present (main effect of stimulus: $F_{1,11}=14.372, p=0.0030$ ) and this effect was particularly apparent in the interaction zone (interaction between zone and stimulus: $F_{2,22}=4.584$; $p=0.0217$, post hoc comparisons: "interaction zone"-"no target" session vs. "interaction zone"-“social target" session, $p<0.05$ ). 
The stress procedure did not affect either the latency to the first episode of risk assessment (main effect of stress condition for stretched-attend posture (SAP) behavior: $F_{1,11}=0.586, p=0.4602$, see Figure $4 \mathrm{C})$ or freezing $\left(F_{1,11}=0.915, p=0.3592\right.$, see Figure 4D). By contrast, regardless of the stress procedure, the presence of an aggressive CD-1 male mouse reduced the latency to freeze in all subjects (main effect of stimulus: $F_{1,11}=10.409, p=0.0081$, data not shown in figure) and increased both frequency and duration of this behavior particularly in the corners (interaction between stimulus and zone: $F_{2,22}=7.095 ; 6.699, p=0.0042 ; 0.0053$, respectively for frequency and duration of freezing; post hoc comparisons: "corners"-"social target" session vs. "corners"-"no target" session, $p<0.01$; "corners" both vs. "center" and "interaction zone", during the "social target" session $p<0.01$ ).

\subsubsection{Long-Term Effects (4 Weeks)}

Four weeks from the end of the SD procedure, when mice were tested in the SAT, an increase in the latency to the first approach to the object and a reduction in the frequency of touching object in all experimental subjects (main effect of stimulus: $F_{1,11}=7.250 ; 5.241, p=0.0209 ; 0.0428$, respectively for latency and frequency) was found. In particular, no effect of the stress experience was found on the latency to approach the cage both in sessions I and II (interaction between stimulus and stress condition $F_{1,11}=0.378, p=0.5513$, see Figure 4E). However, in general, SD mice spent less time in the "interaction zone" compared to the CTRLs (main effect of the stress condition: $F_{1,11}=7.147$, $p=0.0217$, see Figure 4F).

As for locomotor activity, all mice showed an increased frequency of crossings in the interaction zone (main effect of zone: $F_{2,22}=20.650 ; p<0.0001$ ). However, this behavior decreased in this specific portion of the arena from session I to II, i.e., when the CD-1 mouse was introduced in the wire mesh cage (interaction between session and zone for crossings frequency: $F_{2,22}=3.505, p=0.0477$, post hoc comparisons: "interaction zone"-"no target" session vs. "interaction zone"-"social target", $p<0.05$ ).

Overall exposure to the SD procedure reduced the latency to first SAP episode (main effect of stress condition: $F_{1,11}=6.612, p=0.0260$, see Figure $4 \mathrm{G}$ ) as well as the latency to freeze (main effect of stress condition: $F_{1,11}=7.796, p=0.0175$, see Figure $4 \mathrm{H}$ ).

\subsection{Hot Plate (HP) Test}

Twenty-four hours after the end of the social stress (short-term condition), SD mice showed a higher pain threshold than controls, being characterized by lower latency, frequency and duration of hindpaw licking, suggesting overall a SIA profile (main effect of stress: $F_{1,11}=5.302 ; 4.838 ; 5.201$; $p=0.0418$; 0.0500; 0.0435; respectively for latency, frequency and duration, see Figure 5A-C). Interestingly, a similar profile was still evident in SD mice 4 weeks from the end of stress (long-term condition), as shown by a persistent lower frequency of hindpaw licking (main effect of stress: $F_{1,11}=6.769 ; p=0.0246$, see Figure 5E) while no difference was found as for latency and duration (main effect of stress: $F_{1,11}=2.187 ; 1.118, p=0.1673 ; 0.3130$, respectively for latency and duration, see Figure 5D,F). CTRL and SD mice did not differ in forepaw licking behavior either when tested $24 \mathrm{~h}$ or 4 weeks following the end of the stressful procedure (main effect of stress: $24 \mathrm{~h}$, $F_{1,11}=0.525 ; 0.880 ; 0.243, p=0.4840 ; 0.3683 ; 0.6318$, respectively for latency, frequency and duration; 4 weeks, $F_{1,11}=2.015 ; 0.035 ; 0.128, p=0.1834 ; 0.8544 ; 0.7275$, respectively for latency, frequency and duration). 
Figure 5. Hot Plate (HP) Test. Twenty-four h after the end of stress, SD mice were characterized by an increase in pain threshold (SIA) showing a higher latency (A) and a reduced frequency $(\mathbf{B})$ and duration $(\mathbf{C})$ to perform hindpaw licking. A SIA profile was still apparent 4 weeks after the end of the stress procedure, when SD mice performed hindpaw licking with a lower frequency than CTRLs (E). No effect was observed as for the latency (D) and duration (F). Data are presented as mean +S.E.M. $n=7$ (SD) and 6 (CTRL). $* p<0.05 . * * p<0.01$. Subjects were tested only once either at $24 \mathrm{~h}$ or after 4 weeks after the end of SD.

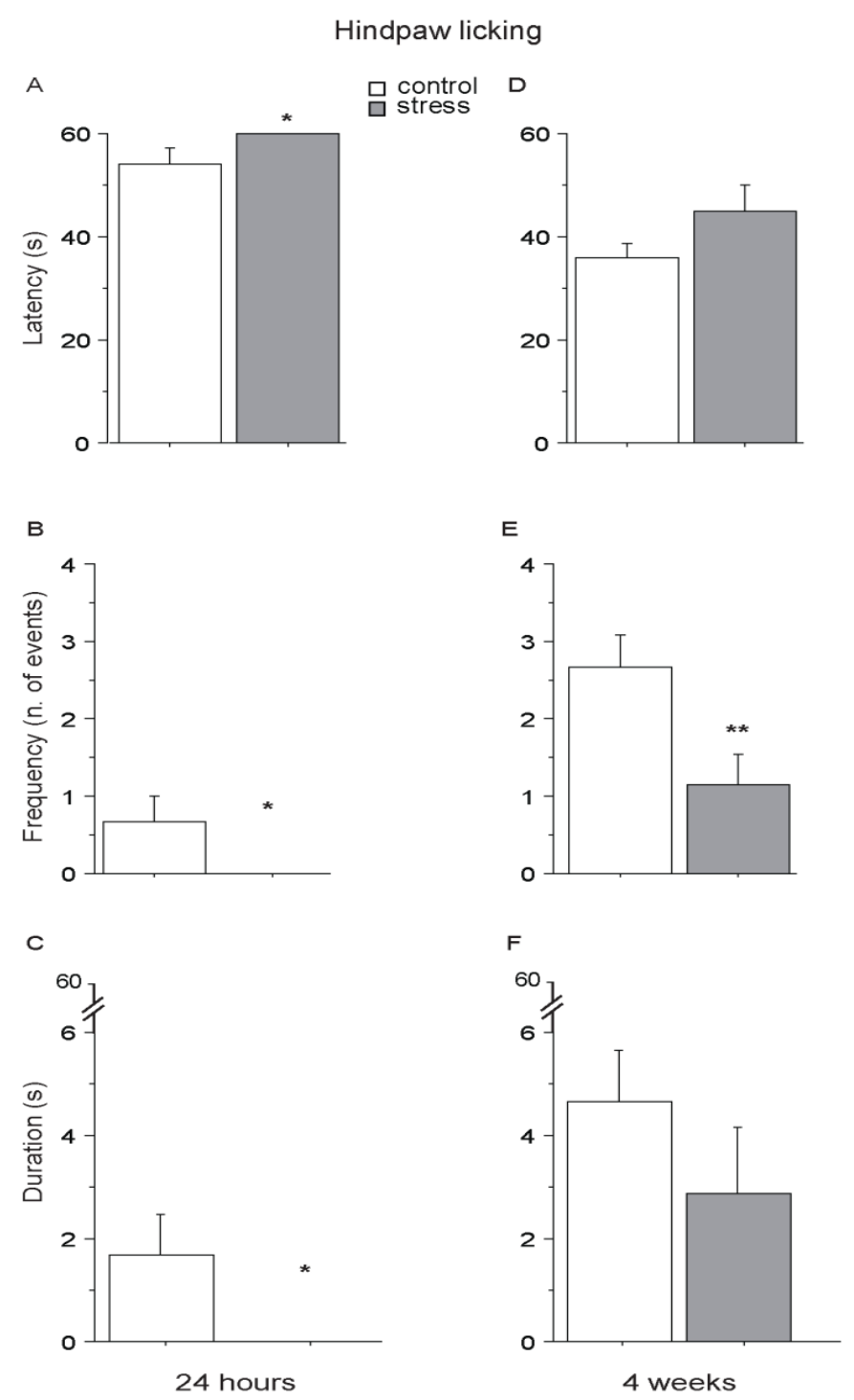

Results from this study show that a chronic social stress paradigm affects the emotional profile of $\mathrm{C} 57 \mathrm{BL} / 6 \mathrm{~J}$ adult male mice as well as their nociceptive response to stressful stimuli. In addition, they provide evidence for SIA as a specific and adaptive biological mechanism with persistent long-term effects that might be elicited and/or reinforced by exposure to stressful stimuli with a similar salience as those previously experienced.

These data suggest that SIA might be useful to assess long-lasting physiological effects of stress-induced vulnerability, such as those characterizing human psychopathological conditions. 
As shown by the increase in corticosterone levels $30 \mathrm{~min}$ following the onset of stress, experimental subjects do not show a significant habituation to the stressful procedure after 10 days of SD, being characterized by a similar neuroendocrine profile on day 1 and on day 10 . This lack of habituation is due to the fact that each day a different social stimulus was used, thus preventing a clear habituation curve, SD representing a significant challenge for the neuroendocrine system. This piece of data was associated to a decrease in body weight (day 10). Adaptive metabolic responses to stress may vary according to the sensitivity to the procedure, duration and intensity of the stressful experience as well as to the mouse strain tested [45]. In our stress paradigm, C57BL/6J subjects, apart from the physical interaction with aggressors, were also in a continuous/chronic visual and olfactive contact with CD-1 subjects during the entire 10-day procedure. A decrease in body weight has been described to result from exposure to chronic social stress [46-48]. In line with these data, our results might indicate a catabolic effect of the daily exposure to the aggressive mouse. Alternatively, although we did not measure it, it is possible that mice undergoing SD were characterized by a decrease in food consumption during the stress administration.

In the SAT, SD subjects were characterized by a higher latency to approach the wire mesh cage in the presence of the CD-1 aggressor (touching object) soon after the end of stress ( $24 \mathrm{~h}$ ). This is a clear indication that the SD procedure was effective in eliciting avoidance of a social target that had acquired salience as a threatening stimulus. Differently from previous studies [16], we did not find a difference in the "time spent in the interaction zone" at this time point neither in the SD nor in the CTRL group. As pointed out by Golden and co-workers, recent evidence suggests that C57BL/6J mice appear to be less susceptible to stress when compared with other strains [49,50]. In addition, these authors argue that in this test "a social interaction ratio equal to 1 , in which equal time is spent in the presence versus absence of a social target, has been used as the threshold for dividing defeated mice into the susceptible and resilient categories [16,48]. Control C57BL/6J mice show a strong tendency to spend greater than or equal amounts of time in the interaction zone in each session. Mice below this criterion are grouped as susceptible, whereas mice above are grouped as resilient" [51]. Therefore, we cannot exclude that by using a larger number of experimental subjects we could have identified subgroups of mice "resilient" or "vulnerable" to the SD procedure. No change was observed overall in the general emotional behavior as we found no difference in the latency to freeze and to perform risk assessment behaviors $(S A P)$.

The higher latency to approach the social target was not found at 4 weeks. However, at this time point, SD mice displayed a general avoidance for the interaction zone, regardless of the presence of the social target, and this was associated with an overall enhancement in their emotional profile as shown by the reduced latency to perform $S A P$ and to freeze upon introduction in the arena. As for freezing, this behavior can be considered as a component of the classic anxiogenic pattern as assessed both in rats and mice as well as a measure of associative fear intensity [52,53]; therefore, we suggest that the behavioral profile observed 4 weeks from the end of SD likely reflects the long-term effects of the social defeat-related stressful experience. This result is further strengthened by the persistence of SIA only in mice that underwent chronic SD stress and up to 4 weeks (increased hindpaw licking frequency).

Indeed, a further main stress-induced change in the physiology of SD mice is the increase in pain threshold, as measured in the hot plate test. This alteration in nociception, also known as SIA, was 
observed not only under acute/short-term (24 h) conditions but also, and more intriguingly, up until 4 weeks from the end of the SD procedure. In the acute situation, the stress-induced analgesic profile was associated with behaviors indicative of social anxiety/avoidance (increased latency to touching object) while, in the long-term, with an overall increase in emotionality (decreased latency to freeze and to perform $S A P$ ). It is interesting to note that the assessment of nociceptive threshold was performed following the SAT i.e., after the exposure of the experimental subjects to a social stimulus (unfamiliar CD-1 mouse) which had been associated with a stressful/traumatic experience (chronic defeat). Although on the one hand the SAT required the animal to come into contact with the aggressive CD-1 mouse in a different context from the SD procedure - and thus we cannot refer to contextual memory [54] in this case - on the other hand, as pointed out by Berton and co-workers, in defeated mice an associative process is required for a social target to progressively acquire salience as a threatening stimulus [16]. Therefore, re-exposure (without physical contact) to an aggressive mouse was able to induce analgesia, as revealed in the hot plate test. Worth noticing, very few studies have investigated the long-term effects of changes in thermal pain sensitivity as a result of stress exposure. As an example, Zhang and co-workers recently found a decrease in the nociceptive threshold (both thermal and mechanical) of Sprague-Dawley rats up to 21 days following the end of heterotypic stress consisting of the exposure of experimental subjects to restraint stress, forced swimming, diethyl ether and prolonged social isolation [55]. However, to our knowledge, this is the first time that such a long-term effect of SIA is reported in mice following the exposure to a repeated homotipic stressor such as SD.

It has been suggested that SD stress might be used as a reliable procedure to model PTSD for its relevance in reproducing some of the symptoms characterizing this pathology or some co-morbid disorders often associated to it [16,52]. Psychiatric pathologies in which stressful/traumatic events play a main role in their etiology, such as PTSD and BPD, are characterized by common stress-induced features such as intrusive memories and dissociative states involving also numbing and SIA $[27,56]$. Although the presence of persistent chronic pain has been often reported in people affected by PTSD [57-59], SIA in these patients appears as a specific response to the re-exposure to a stimulus resembling the original trauma $[25,26]$. A well-established relationship links pain sensitivity endogenous opioids and the SIA $[60,61]$ and a great body of evidence reports specific changes in this biological system as a result of chronic stress [62] and severe physical or psychological traumas [63-65]. In addition, increased pain tolerance has been reported in individuals who self-injure [66] (an aberrant behavior also observed in abused children [67-69]). Likewise, in PTSD patients the secretion of endogenous opioids, as a result of re-exposure to a stimulus resembling the traumatic stress (combat videotapes), was indirectly measured as naloxone reversible anesthesia [25,26]. In a very recent and interesting review, Naomi Eisenberger suggests that "the experiences of social pain-the painful feelings associated with social disconnection-rely on some of the same neurobiological substrates that underlie experiences of physical pain" sharing therefore common signaling pathways leading to the modulation of nociceptive threshold throughout life as fine adaptation to emotional life events [70]. Indeed, chronic pain has been also found to be co-morbid with anxiety and depression [71] in a causeeffect vicious circle and recent evidence reports pain relief in a mouse model of stress as a result of chronic treatment with the selective serotonin reuptake inhibitor fluoxetine (an antidepressant able to reverse anxiety/depressive-like state) [72]. Interestingly, Berton and co-workers found that chronic 
administration of either fluoxetine or imipramine improved social interaction in mice undergoing SD stress, suggesting a link between neuronal pathways involved in the circuitry of reward and sociality [16]; similarly it has been proposed that common pathways involving pain and reward systems might have evolved to support complex social experiences [70,73-76]. Thus, an intriguing hypothesis is that pain, reward and sociality might share common basic mechanisms playing a main role in the etiology, onset and progression of psychiatric disorders.

\section{Conclusions}

Taken together, our data show that the SD stress paradigm is able to induce ethologically and ecologically relevant forms of persistent emotional disruption associated with short- and long-term changes in the nociceptive threshold. In addition, they reinforce previous evidence for SIA as a specific and adaptive biological mechanism with persistent long-term effects that might be elicited and/or reinforced by the exposure to stressful stimuli with similar salience as those previously experienced.

Taking into account all the limitations of our study, also related to the small number of experimental subjects and physiological parameters evaluated, these data suggest that SIA might represent a good physiological response to assess the long-lasting effects of stress-induced vulnerability such as that characterizing human psychopathological conditions.

Indeed, strong epidemiological evidence relates changes in pain perception with disorder of mood and anxiety. In particular, PTSD is associated with significantly elevated prevalence of chronic pain $[57,58]$. Despite this feature, SIA in PTSD patients appears as a specific response to the re-exposure to a stressful stimulus resembling the original trauma [25,26,77]. By contrast, BPD has been associated with reduced pain sensitivity both under stress and non-stress conditions in humans [78-80].

Future studies should further characterize changes in pain sensitivity in animal models of stress in order to design relevant preclinical studies of specific human psychopathologies. Progress in understanding the pathophysiology of human psychopathologies would greatly benefit from preclinical studies incorporating SIA as a physiological marker of stress.

\section{Acknowledgments}

We kindly acknowledge Carla Raggi for technical support in the preparation of this manuscript. Funding for this study was provided by the Italian Ministry of Health (Italia-USA Project) Fasc.11US/14/1 to E.A.

\section{Author Contributions}

A. Berry and V. Bellisario wrote the manuscript; F. Cirulli, E. Alleva and A. Berry designed the experimental protocol; F. Cirulli and E. Alleva, revised the manuscript, V. Bellisario, S. Capoccia and N. Francia performed the experiments.

\section{Conflicts of Interest}

All authors declare that no conflict of interests, financial or otherwise, exists with regard to this research. 


\section{References}

1. Anisman, H.; Merali, Z.; Stead, J.D. Experiential and genetic contributions to depressive- and anxiety-like disorders: Clinical and experimental studies. Neurosci. Biobehav. Rev. 2008, 32, $1185-1206$.

2. Cirulli, F.; Alleva, E. The NGF saga: From animal models of psychosocial stress to stress-related psychopathology. Front Neuroendocrinol. 2009, 30, 379-395.

3. Cryan, J.F.; Holmes, A. The ascent of mouse: Advances in modelling human depression and anxiety. Nat. Rev. Drug Discov. 2005, 4, 775-790.

4. Koolhaas, J.M.; de Boer, S.F.; Buwalda, B.; van Reenen, K. Individual variation in coping with stress: A multidimensional approach of ultimate and proximate mechanisms. Brain Behav. Evol. 2007, 70, 218-226.

5. Miczek, K.A.; de Wit, H. Challenges for translational psychopharmacology research—Some basic principles. Psychopharmacology (Berl.) 2008, 199, 291-301.

6. Koolhaas, J.M.; de Boer, S.F.; de Rutter, A.J.; Meerlo, P.; Sgoifo, A. Social stress in rats and mice. Acta Physiol. Scand Suppl. 1997, 640, 69-72.

7. Tamashiro, K.L.; Nguyen, M.M.; Sakai, R.R. Social stress: From rodents to primates. Front Neuroendocrinol. 2005, 26, 27-40.

8. Willner, P. The validity of animal models of depression. Psychopharmacology (Berl.) 1984, 83, 1-16.

9. Berry, A.; Bellisario, V.; Capoccia, S.; Tirassa, P.; Calza, A.; Alleva, E.; Cirulli, F. Social deprivation stress is a triggering factor for the emergence of anxiety- and depression-like behaviours and leads to reduced brain BDNF levels in C57BL/6J mice. Psychoneuroendocrinology 2012, 37, $762-772$.

10. Kudryavtseva, N.N.; Bakshtanovskaya, I.V.; Koryakina, L.A. Social model of depression in mice of C57BL/6J strain. Pharmacol. Biochem. Behav. 1991, 38, 315-320.

11. Sheridan, J.F.; Stark, J.L.; Avitsur, R.; Padgett, D.A. Social disruption, immunity, and susceptibility to viral infection. Role of glucocorticoid insensitivity and NGF. Ann. N. Y. Acad. Sci. 2000, 917, 894-905.

12. Vialou, V.; Robison, A.J.; Laplant, Q.C.; Covington, H.E., 3rd.; Dietz, D.M.; Ohnishi, Y.N.; Mouzon, E.; Rush, A.J., 3rd; Watts, E.L.; Wallace, D.L.; et al. DeltaFosB in brain reward circuits mediates resilience to stress and antidepressant responses. Nat. Neurosci. 2010, 13, 745-752.

13. Bartolomucci, A.; Palanza, P.; Sacerdote, P.; Panerai, A.E.; Sgoifo, A.; Dantzer, R.; Parmigiani, S. Social factors and individual vulnerability to chronic stress exposure. Neurosci. Biobehav. Rev. 2005, 29, 67-81.

14. Beitia, G.; Garmendia, L.; Azpiroz, A.; Vegas, O.; Brain, P.F.; Arregi, A. Time-dependent behavioral, neurochemical, and immune consequences of repeated experiences of social defeat stress in male mice and the ameliorative effects of fluoxetine. Brain Behav. Immun. 2005, 19, 530-539.

15. Fuchs, E. Social stress in tree shrews as an animal model of depression: An example of a behavioral model of a CNS disorder. CNS Spectr. 2005, 10, 182-190. 
16. Berton, O.; McClung, C.A.; Dileone, R.J.; Krishnan, V.; Renthal, W.; Russo, S.J.; Graham, D.; Tsankova, N.M.; Bolanos, C.A.; Rios, M.; et al. Essential role of BDNF in the mesolimbic dopamine pathway in social defeat stress. Science 2006, 311, 864-868.

17. Brown, G.W. Social roles, context and evolution in the origins of depression. J. Health Soc. Behav. 2002, 43, 255-276.

18. Tidey, J.W.; Miczek, K.A. Acquisition of cocaine self-administration after social stress: Role of accumbens dopamine. Psychopharmacology (Berl.) 1997, 130, 203-212.

19. Avgustinovich, D.F.; Kovalenko, I.L.; Kudryavtseva, N.N. A model of anxious depression: Persistence of behavioral pathology. Neurosci. Behav. Physiol. 2005, 35, 917-924.

20. Huhman, K.L.; Solomon, M.B.; Janicki, M.; Harmon, A.C.; Lin, S.M.; Israel, J.E.; Jasnow, A.M. Conditioned defeat in male and female Syrian hamsters. Horm. Behav. 2003, 44, 293-299.

21. Siegfried, B.; Frischknecht, H.R.; Waser, P.G. Defeat, learned submissiveness, and analgesia in mice: Effect of genotype. Behav. Neural. Biol. 1984, 42, 91-97.

22. Koolhaas, J.M.; Meerlo, P.; de Boer, S.F.; Strubbe, J.H.; Bohus, B. The temporal dynamics of the stress response. Neurosci. Biobehav. Rev. 1997, 21, 775-782.

23. Meerlo, P.; de Boer, S.F.; Koolhaas, J.M.; Daan, S.; van den Hoofdakker, R.H. Changes in daily rhythms of body temperature and activity after a single social defeat in rats. Physiol. Behav. 1996, 59, 735-739.

24. Ruis, M.A.; te Brake, J.H.; Buwalda, B.; de Boer, S.F.; Meerlo, P.; Korte, S.M.; Blokhuis, H.J.; Koolhaas, J.M. Housing familiar male wildtype rats together reduces the long-term adverse behavioural and physiological effects of social defeat. Psychoneuroendocrinology 1999, 24, 285-300.

25. Van der Kolk, B.A.; Greenberg, M.S.; Orr, S.P.; Pitman, R.K. Endogenous opioids, stress induced analgesia, and posttraumatic stress disorder. Psychopharmacol. Bull. 1989, 25, 417-421.

26. Pitman, R.K.; van der Kolk, B.A.; Orr, S.P.; Greenberg, M.S. Naloxone-reversible analgesic response to combat-related stimuli in posttraumatic stress disorder. A pilot study. Arch. Gen. Psychiatry 1990, 47, 541-544.

27. Ludascher, P.; Valerius, G.; Stiglmayr, C.; Mauchnik, J.; Lanius, R.A.; Bohus, M.; Schmahl, C. Pain sensitivity and neural processing during dissociative states in patients with borderline personality disorder with and without comorbid posttraumatic stress disorder: A pilot study. J. Psychiatry Neurosci. 2010, 35, 177-184.

28. Amir, S.; Brown, Z.W.; Amit, Z. The role of endorphins in stress: Evidence and speculations. Neurosci. Biobehav. Rev. 1980, 4, 77-86.

29. Bodnar, R.J.; Kelly, D.D.; Brutus, M.; Glusman, M. Stress-induced analgesia: Neural and hormonal determinants. Neurosci. Biobehav. Rev. 1980, 4, 87-100.

30. Chance, W.T. Autoanalgesia: Opiate and non-opiate mechanisms. Neurosci. Biobehav. Rev. 1980, $4,55-67$.

31. Lewis, J.W.; Cannon, J.T.; Liebeskind, J.C. Opioid and nonopioid mechanisms of stress analgesia. Science 1980, 208, 623-625.

32. Watkins, L.R.; Mayer, D.J. Organization of endogenous opiate and nonopiate pain control systems. Science 1982, 216, 1185-1192.

33. Butler, R.K.; Finn, D.P. Stress-induced analgesia. Prog. Neurobiol. 2009, 88, 184-202. 
34. Bolles, R.C.; Fanselow, M.S. A perceptual-defensive-recuperative model of fear and pain. Behav. Brain Sci. 1980, 3, 291-301.

35. Miczek, K.A.; Thompson, M.L.; Shuster, L. Opioid-like analgesia in defeated mice. Science 1982, $215,1520-1522$.

36. Rodgers, R.J.; Hendrie, C.A. Social conflict activates status-dependent endogenous analgesic or hyperalgesic mechanisms in male mice: Effects of naloxone on nociception and behaviour. Physiol. Behav. 1983, 30, 775-780.

37. Rodgers, R.J.; Hendrie, C.A.; Waters, A.J. Naloxone partially antagonizes post-encounter analgesia and enhances defensive responding in male rats exposed to attack from lactating conspecifics. Physiol. Behav. 1983, 30, 781-786.

38. Teskey, G.C.; Kavaliers, M.; Hirst, M. Social conflict activates opioid analgesic and ingestive behaviors in male mice. Life Sci. 1984, 35, 303-315.

39. Jackson, R.L.; Maier, S.F.; Coon, D.J. Long-term analgesic effects of inescapable shock and learned helplessness. Science 1979, 206, 91-93.

40. Bartlang, M.S.; Neumann, I.D.; Slattery, D.A.; Uschold-Schmidt, N.; Kraus, D.; Helfrich-Forster, C.; Reber, S.O. Time matters: Pathological effects of repeated psychosocial stress during the active, but not inactive, phase of male mice. J. Endocrinol. 2012, 215, 425-437.

41. Fluttert, M.; Dalm, S.; Oitzl, M.S. A refined method for sequential blood sampling by tail incision in rats. Lab. Anim. 2000, 34, 372-378.

42. Sadler, A.M.; Bailey, S.J. Validation of a refined technique for taking repeated blood samples from juvenile and adult mice. Lab. Anim. 2013, 47, 316-319.

43. Berry, A.; Capone, F.; Giorgio, M.; Pelicci, P.G.; de Kloet, E.R.; Alleva, E.; Minghetti, L.; Cirulli, F. Deletion of the life span determinant p66Shc prevents age-dependent increases in emotionality and pain sensitivity in mice. Exp. Gerontol. 2007, 42, 37-45.

44. Cirulli, F.; Berry, A.; Alleva, E. Intracerebroventricular administration of brain-derived neurotrophic factor in adult rats affects analgesia and spontaneous behaviour but not memory retention in a Morris Water Maze task. Neurosci. Lett. 2000, 287, 207-210.

45. Savignac, H.M.; Finger, B.C.; Pizzo, R.C.; O’Leary, O.F.; Dinan, T.G.; Cryan, J.F. Increased sensitivity to the effects of chronic social defeat stress in an innately anxious mouse strain. Neuroscience 2011, 192, 524-536.

46. Reber, S.O.; Obermeier, F.; Straub, R.H.; Falk, W.; Neumann, I.D. Chronic intermittent psychosocial stress (social defeat/overcrowding) in mice increases the severity of an acute DSS-induced colitis and impairs regeneration. Endocrinology 2006, 147, 4968-4976.

47. Savignac, H.M.; Hyland, N.P.; Dinan, T.G.; Cryan, J.F. The effects of repeated social interaction stress on behavioural and physiological parameters in a stress-sensitive mouse strain. Behav. Brain Res. 2011, 216, 576-584.

48. Krishnan, V.; Han, M.H.; Graham, D.L.; Berton, O.; Renthal, W.; Russo, S.J.; Laplant, Q.; Graham, A.; Lutter, M.; Lagace, D.C.; et al. Molecular adaptations underlying susceptibility and resistance to social defeat in brain reward regions. Cell 2007, 131, 391-404.

49. Razzoli, M.; Carboni, L.; Andreoli, M.; Ballottari, A.; Arban, R. Different susceptibility to social defeat stress of BalbC and C57BL6/J mice. Behav. Brain Res. 2010, 216, 100-108. 
50. Razzoli, M.; Carboni, L.; Andreoli, M.; Michielin, F.; Ballottari, A.; Arban, R. Strain-specific outcomes of repeated social defeat and chronic fluoxetine treatment in the mouse. Pharmacol. Biochem. Behav. 2010, 97, 566-576.

51. Golden, S.A.; Covington, H.E., 3rd.; Berton, O.; Russo, S.J. A standardized protocol for repeated social defeat stress in mice. Nat. Protoc. 2011, 6, 1183-1191.

52. Hammamieh, R.; Chakraborty, N.; De Lima, T.C.; Meyerhoff, J.; Gautam, A.; Muhie, S.; D’Arpa, P.; Lumley, L.; Carroll, E.; Jett, M. Murine model of repeated exposures to conspecific trained aggressors simulates features of post-traumatic stress disorder. Behav. Brain Res. 2012, 235, 55-66.

53. Litvin, Y.; Murakami, G.; Pfaff, D.W. Effects of chronic social defeat on behavioral and neural correlates of sociality: Vasopressin, oxytocin and the vasopressinergic V1b receptor. Physiol. Behav. 2011, 103, 393-403.

54. Girardi, C.E.; Tiba, P.A.; Llobet, G.B.; Levin, R.; Abilio, V.C.; Suchecki, D. Contextual exploration previous to an aversive event predicts long-term emotional consequences of severe stress. Front. Behav. Neurosci. 2013, 7, doi:10.3389/fnbeh.2013.00134.

55. Zhang, Y.; Gandhi, P.R.; Standifer, K.M. Increased nociceptive sensitivity and nociceptin/orphanin FQ levels in a rat model of PTSD. Mol. Pain 2012, 8, doi:10.1186/1744-8069-8-76.

56. Ludascher, P.; Bohus, M.; Lieb, K.; Philipsen, A.; Jochims, A.; Schmahl, C. Elevated pain thresholds correlate with dissociation and aversive arousal in patients with borderline personality disorder. Psychiatry Res. 2007, 149, 291-296.

57. Beckham, J.C.; Crawford, A.L.; Feldman, M.E.; Kirby, A.C.; Hertzberg, M.A.; Davidson, J.R.; Moore, S.D. Chronic posttraumatic stress disorder and chronic pain in Vietnam combat veterans. J. Psychosom. Res. 1997, 43, 379-389.

58. Shipherd, J.C.; Keyes, M.; Jovanovic, T.; Ready, D.J.; Baltzell, D.; Worley, V.; Gordon-Brown, V.; Hayslett, C.; Duncan, E. Veterans seeking treatment for posttraumatic stress disorder: What about comorbid chronic pain? J. Rehabil. Res. Dev. 2007, 44, 153-166.

59. Asmundson, G.J.; Katz, J. Understanding the co-occurrence of anxiety disorders and chronic pain: State-of-the-art. Depress Anxiety 2009, 26, 888-901.

60. Rubinstein, M.; Mogil, J.S.; Japon, M.; Chan, E.C.; Allen, R.G.; Low, M.J. Absence of opioid stress-induced analgesia in mice lacking beta-endorphin by site-directed mutagenesis. Proc. Natl. Acad. Sci. USA 1996, 93, 3995-4000.

61. Yamada, K.; Nabeshima, T. Stress-induced behavioral responses and multiple opioid systems in the brain. Behav. Brain Res. 1995, 67, 133-145.

62. Pike, J.L.; Smith, T.L.; Hauger, R.L.; Nicassio, P.M.; Patterson, T.L.; McClintick, J.; Costlow, C.; Irwin, M.R. Chronic life stress alters sympathetic, neuroendocrine, and immune responsivity to an acute psychological stressor in humans. Psychosom. Med. 1997, 59, 447-457.

63. Birrell, A.M.; Heffernan, S.J.; Ansselin, A.D.; McLennan, S.; Church, D.K.; Gillin, A.G.; Yue, D.K. Functional and structural abnormalities in the nerves of type I diabetic baboons: Aminoguanidine treatment does not improve nerve function. Diabetologia 2000, 43, 110-116.

64. Van Goozen, S.H.; van den Ban, E.; Matthys, W.; Cohen-Kettenis, P.T.; Thijssen, J.H.; van Engeland, H. Increased adrenal androgen functioning in children with oppositional defiant disorder: A comparison with psychiatric and normal controls. J. Am. Acad. Child Adolesc. Psychiatry 2000, 39, 1446-1451. 
65. Bremner, J.D. Long-term effects of childhood abuse on brain and neurobiology. Child Adolesc. Psychiatr. Clin. North Am. 2003, 12, 271-292.

66. Claes, L.; Vandereycken, W.; Vertommen, H. Pain experience related to self-injury in eating disorder patients. Eat. Behav. 2006, 7, 204-213.

67. Zanarini, M.C.; Yong, L.; Frankenburg, F.R.; Hennen, J.; Reich, D.B.; Marino, M.F.; Vujanovic, A.A. Severity of reported childhood sexual abuse and its relationship to severity of borderline psychopathology and psychosocial impairment among borderline inpatients. J. Nerv. Ment. Dis. 2002, 190, 381-387.

68. Gratz, K.L.; Hepworth, C.; Tull, M.T.; Paulson, A.; Clarke, S.; Remington, B.; Lejuez, C.W. An experimental investigation of emotional willingness and physical pain tolerance in deliberate self-harm: The moderating role of interpersonal distress. Compr. Psychiatry 2011, 52, 63-74.

69. Joyce, P.R.; McKenzie, J.M.; Mulder, R.T.; Luty, S.E.; Sullivan, P.F.; Miller, A.L.; Kennedy, M.A. Genetic, developmental and personality correlates of self-mutilation in depressed patients. Aust. N. Z. J. Psychiatry 2006, 40, 225-229.

70. Eisenberger, N.I. The pain of social disconnection: examining the shared neural underpinnings of physical and social pain. Nat. Rev. Neurosci. 2012, 13, 421-434.

71. Moeller-Bertram, T.; Keltner, J.; Strigo, I.A. Pain and post traumatic stress disorder-Review of clinical and experimental evidence. Neuropharmacology 2012, 62, 586-597.

72. Hache, G.; Guiard, B.P.; le Dantec, Y.; Orvoen, S.; David, D.J.; Gardier, A.M.; Coudore, F. Antinociceptive effects of fluoxetine in a mouse model of anxiety/depression. Neuroreport 2012, 23, 525-529.

73. Moll, J.; Krueger, F.; Zahn, R.; Pardini, M.; de Oliveira-Souza, R.; Grafman, J. Human fronto-mesolimbic networks guide decisions about charitable donation. Proc. Natl. Acad. Sci. USA 2006, 103, 15623-15628.

74. Keysers, C.; Kaas, J.H.; Gazzola, V. Somatosensation in social perception. Nat. Rev. Neurosci. 2010, 11, 417-428.

75. Lamm, C.; Decety, J.; Singer, T. Meta-analytic evidence for common and distinct neural networks associated with directly experienced pain and empathy for pain. Neuroimage 2011, 54, 2492-2502.

76. Inagaki, T.K.; Eisenberger, N.I. Neural correlates of giving support to a loved one. Psychosom. Med. 2012, 74, 3-7.

77. Mickleborough, M.J.; Daniels, J.K.; Coupland, N.J.; Kao, R.; Williamson, P.C.; Lanius, U.F.; Hegadoren, K.; Schore, A.; Densmore, M.; Stevens, T.; Lanius, R.A. Effects of trauma-related cues on pain processing in posttraumatic stress disorder: An fMRI investigation. J. Psychiatry Neurosci. 2011, 36, 6-14.

78. Schmahl, C.; Bohus, M.; Esposito, F.; Treede, R.D.; Di Salle, F.; Greffrath, W.; Ludaescher, P.; Jochims, A.; Lieb, K.; Scheffler, K.; et al. Neural correlates of antinociception in borderline personality disorder. Arch. Gen. Psychiatry 2006, 63, 659-667.

79. Russ, M.J.; Roth, S.D.; Lerman, A.; Kakuma, T.; Harrison, K.; Shindledecker, R.D.; Hull, J.; Mattis, S. Pain perception in self-injurious patients with borderline personality disorder. Biol. Psychiatry 1992, 32, 501-511. 
80. Bohus, M.; Limberger, M.; Ebner, U.; Glocker, F.X.; Schwarz, B.; Wernz, M.; Lieb, K. Pain perception during self-reported distress and calmness in patients with borderline personality disorder and self-mutilating behavior. Psychiatry Res. 2000, 95, 251-260.

(C) 2014 by the authors; licensee MDPI, Basel, Switzerland. This article is an open access article distributed under the terms and conditions of the Creative Commons Attribution license (http://creativecommons.org/licenses/by/3.0/). 